\title{
Mn-induced multiple magnetic ground states in $\operatorname{Sr}_{3}\left(\operatorname{Ru}_{1-x} \mathbf{M n}_{x}\right)_{2} \mathbf{O}_{7}$
}

\author{
Roshan Nepal $\odot,{ }^{1}$ Lingyi Xing, ${ }^{1}$ Arno Meingast $\odot,{ }^{2}$ and Rongying Jin $\oplus^{1,3, *}$ \\ ${ }^{1}$ Department of Physics and Astronomy, Louisiana State University, Baton Rouge, Louisiana 70803, USA \\ ${ }^{2}$ Thermo Fisher Scientific, 5651 GH De Schakel, Eindhoven, Netherlands \\ ${ }^{3}$ Center for Experimental Nanoscale Physics, Department of Physics and Astronomy, University of South Carolina, \\ Columbia, South Carolina 29208, USA
}

(Received 29 July 2021; revised 11 November 2021; accepted 15 November 2021; published 16 December 2021)

\begin{abstract}
Transition-metal oxides provide an excellent platform to generate ground states by tuning the various degrees of freedom via stimuli such as chemical doping. By investigating the electrical, magnetic, and thermodynamic properties of several compositions of the bilayered $\mathrm{Sr}_{3}\left(\mathrm{Ru}_{1-x} \mathrm{Mn}_{x}\right)_{2} \mathrm{O}_{7}$ with $x \geqslant 0.36$, we complete its $x-T$ phase diagram and unveil a spin-glass regime. Upon Mn doping, the ground state of the system changes from the shortrange antiferromagnetically (AFM) coupled metal $(0 \leqslant x \leqslant 0.05)$ to the E-type AFM-ordered insulator $(0.05<$ $x \leqslant 0.20)$ to the spin-glass insulator $(0.20<x \leqslant 0.66)$ to the canted AFM-ordered insulator $(x \sim 1.0)$. The complex evolution of the magnetic ground state results from the competition between AFM and ferromagnetic (FM) interactions. The peculiar spin-glass state between two long-range magnetically ordered states implies the significance of spin disorder induced by the intermixing of $\mathrm{Mn}$ and $\mathrm{Ru}$ ions with the lowest transition temperature for $\mathrm{Ru}: \mathrm{Mn} \sim 1: 1(x \sim 0.5)$.
\end{abstract}

DOI: 10.1103/PhysRevResearch.3.043183

\section{INTRODUCTION}

Correlated transition-metal oxides (TMOs) have attracted considerable interest due to the abundance of exciting physical phenomena arising from the strong interactions between charge, lattice, orbital, and spin degrees of freedom. Among TMOs, the Ruddleson-Popper strontium ruthenate series with the general formula $\mathrm{Sr}_{n+1} \mathrm{Ru}_{n} \mathrm{O}_{3 n+1}(n=1,2, \ldots \infty)$ is widely known for intriguing physical properties such as Mott metal-insulator transition (MIT), unconventional superconductivity, inherently competing magnetic interactions, and field-induced quantum criticality [1-5]. In this series, the bilayered $\mathrm{Sr}_{3} \mathrm{Ru}_{2} \mathrm{O}_{7}(n=2)$ crystallizes in a slightly distorted tetragonal lattice structure, as shown in Fig. 1(a), that consists of two layers of $\mathrm{RuO}_{6}$ octahedra planes stacked along the $c$ axis. It is a paramagnetic metal developing short-range antiferromagnetic (AFM) correlation $<\sim 18 \mathrm{~K}[1,6]$. However, it has been reported to possess ferromagnetic (FM) fluctuations as well $[7,8]$. The presence of both AFM and FM interactions results in a fragile magnetic ground state that shows remarkable response to external stimuli such as magnetic field, pressure, and chemical substitution [1,3,9], for example, the application of magnetic field results in the second-order metamagnetic phase transition [3,4]. By pushing the temperature $T \rightarrow 0 \mathrm{~K}$, the field-induced quantum critical point $(\mathrm{QCP})$ can be reached, and the electronic nematicity is

\footnotetext{
*rjin@mailbox.sc.edu

Published by the American Physical Society under the terms of the Creative Commons Attribution 4.0 International license. Further distribution of this work must maintain attribution to the author(s) and the published article's title, journal citation, and DOI.
}

seen around the QCP [10-12]. Similarly, the application of hydrostatic pressure can stabilize the FM interaction in the system $[6,13]$.

Competing interactions have been unveiled through chemical doping in either the $\mathrm{Sr}$ or $\mathrm{Ru}$ site. While isovalent doping, a partial substitution of $\mathrm{Sr}^{2+}$ by smaller $\mathrm{Ca}^{2+}$ results in spin-glass (SG) and AFM (A-type) insulating states in $\left(\mathrm{Sr}_{1-x} \mathrm{Ca}_{x}\right)_{3} \mathrm{Ru}_{2} \mathrm{O}_{7}$ [14-17]. As the physical properties of $\mathrm{Sr}_{3} \mathrm{Ru}_{2} \mathrm{O}_{7}$ are determined by $\mathrm{Ru}$ bands [18], a partial substitution of $\mathrm{Ru}$ by other transition metals can dramatically tip the inherent interactions in $\mathrm{Sr}_{3} \mathrm{Ru}_{2} \mathrm{O}_{7}$, resulting in a rich phase diagram $[1,19]$. For example, introducing $5 d$ Ir leads to initially an AFM metallic phase $(0.4<x<0.6)$ and eventually an insulating canted AFM state $(x>0.6)$ in $\mathrm{Sr}_{3}\left(\mathrm{Ru}_{1-x} \mathrm{Ir}_{x}\right)_{2} \mathrm{O}_{7}$ [19]. Such a result is unexpected, given that Ir has more extended $d$ orbitals with more electrons than that of Ru. On the other hand, when Ru ions are substituted by $3 d \mathrm{Mn}$, an equally complex evolution of the magnetic and electronic ground states are observed in $\mathrm{Sr}_{3}\left(\mathrm{Ru}_{1-x} \mathrm{Mn}_{x}\right)_{2} \mathrm{O}_{7}$ [1]. Upon Mn doping $(x)$, the electronic ground state becomes insulating with the MIT temperature $\left(T_{\mathrm{MIT}}\right)$ increasing with $x$. Simultaneously, the introduction of Mn drives the doped system into long-range AFM ordering at $T_{\mathrm{N}}$ with $T_{\mathrm{N}}<T_{\mathrm{MIT}}$. Unlike $T_{\mathrm{MIT}}$, which increases with increasing $x, T_{\mathrm{N}}$ varies nonmonotonically with $x$ in the region $0.05<x<0.20$, revealing a local maximum at $x \sim 0.16$. Such a doping dependence of $T_{\mathrm{N}}$ is extremely intriguing, implying complex interplay between various magnetic interactions. While significant attention has been paid to the low Mn-doping region of this series, information about how the system behaves beyond the long-range AFM order in the region $0.05<x<0.20$ is lacking in the literature. In this paper, by measuring the electrical and magnetic properties of $x=0.36,0.55,0.66$, and 1.0 single crystals, we unveil the magnetic interplay that leads to the 
(a)

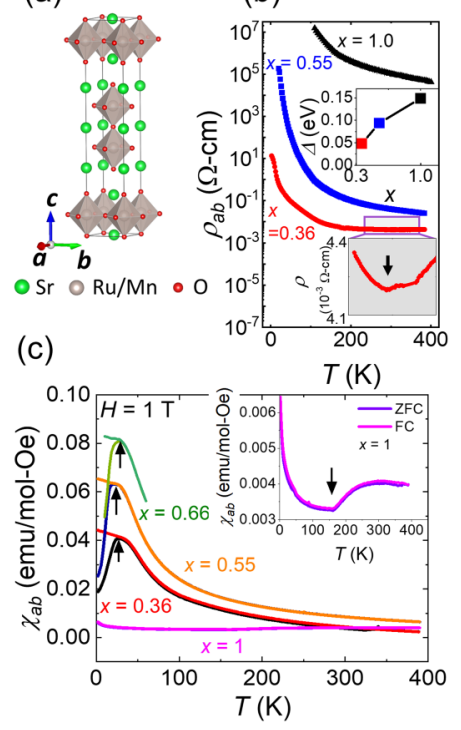

(d)

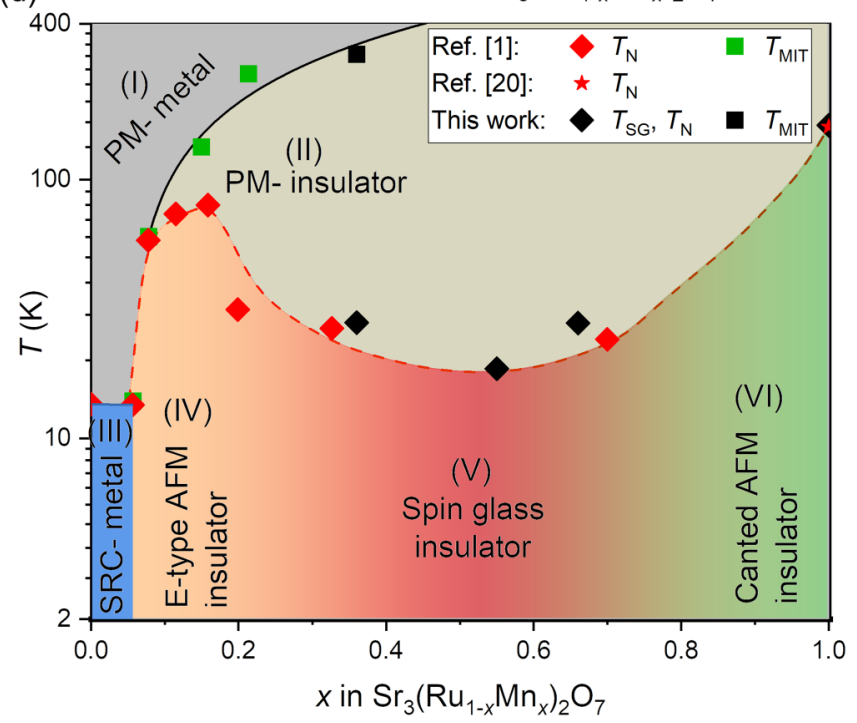

FIG. 1. (a) Crystal structure of $\mathrm{Sr}_{3}\left(\mathrm{Ru}_{1-x} \mathrm{Mn}_{x}\right)_{2} \mathrm{O}_{7}$. (b) Temperature dependence of the in-plane resistivity for $x=0.36,0.55$, and 1.0. Top inset: $x$ dependence of the activation energy estimated via the Arrhenius fitting of the resistivity data. Bottom inset: Enlarged view of the temperature dependence of resistivity for $x=0.36$ between 250 and $375 \mathrm{~K}$ with the metal-insulator transition temperature $T_{\mathrm{MIT}}$ indicated by an arrow. (c) Temperature dependence of the magnetic susceptibility measured under $H=1 \mathrm{~T}$ parallel to the $a b$ plane $\left(\chi_{a b}\right)$ for $x=0.36,0.55$, 0.66, and 1.0. Inset shows the enlarged view of $\chi_{a b}$ for $x=1.0$. (d) Phase diagram of $\operatorname{Sr}_{3}\left(\mathrm{Ru}_{1-x} \mathrm{Mn}_{x}\right)_{2} \mathrm{O}_{7}$. The colored points are taken from Refs. $[1,20]$, and the black points are from this paper.

unusual evolution of the magnetic ground state in the higher Mn-doped region $(x>0.20)$ and complete the phase diagram of $\mathrm{Sr}_{3}\left(\mathrm{Ru}_{1-x} \mathrm{Mn}_{x}\right)_{2} \mathrm{O}_{7}$. Our experimental observations reveal the presence of the SG ground state in a wide doping range with the lowest transition temperature $T_{\mathrm{SG}}$ located at $x \sim 0.5$. As $x$ approaches 1.0, the SG behavior is weakened, while a long-range magnetic order is formed in $\mathrm{Sr}_{3} \mathrm{Mn}_{2} \mathrm{O}_{7}(x=1.0)$ below $T_{\mathrm{N}}=160 \mathrm{~K}$. Our complete magnetic phase diagram covering $0 \leqslant x \leqslant 1$ allows us to understand the underlying physics of $\mathrm{Sr}_{3}\left(\mathrm{Ru}_{1-x} \mathrm{Mn}_{x}\right)_{2} \mathrm{O}_{7}$ thoroughly by considering multiple magnetic interactions.

\section{METHODS}

Single crystals of $\mathrm{Sr}_{3}\left(\mathrm{Ru}_{1-x} \mathrm{Mn}_{x}\right)_{2} \mathrm{O}_{7}$ with several nominal $x_{\text {nominal }}$ values between 0.3 and $1.0\left(x_{\text {nominal }}=0.3,0.6,0.75\right.$, and 1.0) were grown in a floating zone furnace under 0.9 MPa oxygen pressure. The phase purity of the crystals was checked via room-temperature $\mathrm{x}$-ray diffraction (XRD) using a PANalytical Empyrean x-ray diffractometer with $\mathrm{Cu} \mathrm{K} \alpha$ radiation (see Fig. 2). The chemical compositions of the crystals were determined using energy dispersive $\mathrm{x}$-ray spectroscopy (EDS) equipped by FEI Quanta 200 under vacuum environment. The actual $\mathrm{Mn} / \mathrm{Ru}$ ratios of the crystals were found to be $x=0.35,0.55,0.66$, and 1.0 (see Table I); the actual $x$ values are used in this paper. To avoid oxygen deficiency, the as-grown samples were annealed in an oxygen atmosphere at $550-600{ }^{\circ} \mathrm{C}$ for $6 \mathrm{wk}$. The magnetization measurements were performed in a magnetic properties measurement system (MPMS-7 T, Quantum Design), and the electrical transport and specific heat measurements were performed in a physical properties measurement system (PPMS-14 T, Quantum
Design). The high-temperature magnetization measurements were done using a vibrating sample magnetometer option. High-resolution scanning transmission electron microscopy (STEM), elemental electron energy loss spectroscopy (EELS) elemental mapping, and energy dispersive $\mathrm{x}$-ray spectroscopy (EDS) elemental mapping measurements were performed using Thermo Scientific Spectra 300 (S)TEM equipped with $\mathrm{X}$-CFEG. The microscope settings for the STEM images were $200 \mathrm{kV}, \mathrm{C} 2$ aperture of $50 \mu \mathrm{m}, 23$ mrads semiconvergence angle, cathodoluminescence of $320 \mathrm{~mm}$ EFSTEM with indicated magnification of 7.2 MX and beam current of $129 \mathrm{pA}$. For the high-resolution (HR) EELS spectra, GIF Continuum HR was utilized with entrance aperture of $5 \mathrm{~mm}$, dispersion of $1.5 \mathrm{eV} /$ channel, semicollection angle of $73.5 \mathrm{mrads}$, dwell time of $0.005 \mathrm{~s}$ per pixel, total acquisition time of $117 \mathrm{~s}$, and high-loss exposure of $4.95 \mathrm{~ms}$. For EDS mapping, the total acquisition time was $1268 \mathrm{~s}$ with the probe current of $129 \mathrm{pA}$.

\section{RESULTS AND DISCUSSION}

Figure 1(b) shows the temperature dependence of the inplane electrical resistivity $\rho_{a b}$ for $x=0.36,0.55$, and 1.0 samples. Overall, $\rho_{a b}$ increases with increasing $x$ in the entire temperature range measured. For $x=0.36$, upon cooling, $\rho_{a b}$ initially decreases, displaying a metallic behavior until $T_{\mathrm{MIT}} \sim 300 \mathrm{~K}$, below which $\rho_{a b}$ increases sharply as the system enters an insulating state. For $x=0.55$ and 1.0, the system displays an insulating behavior without sign of MIT below $400 \mathrm{~K}$. By fitting the data to the Arrhenius function $\rho_{a b}(T)=\rho_{0} \exp \left(\Delta / 2 k_{B} T\right)$, we find that the activation energy 

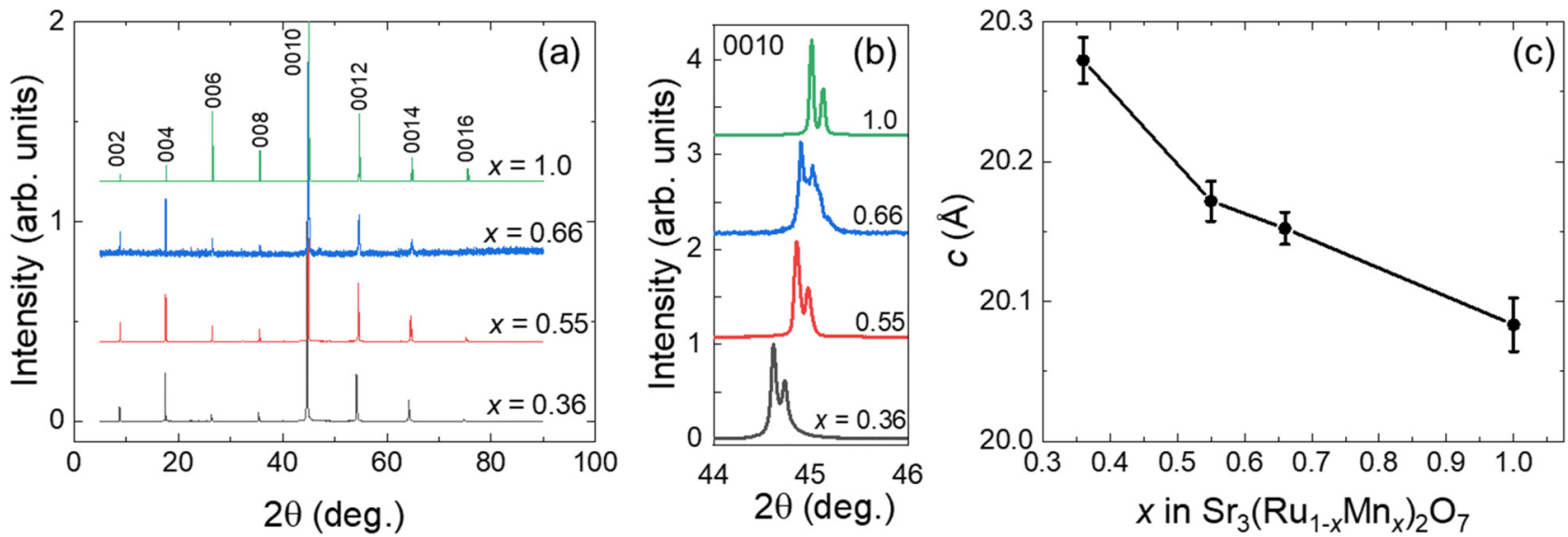

FIG. 2. (a) Room temperature $\mathrm{x}$-ray diffraction (XRD) patterns obtained from the flat ( 00 l) surfaces of $\operatorname{Sr}_{3}\left(\mathrm{Ru}_{1-x} \mathrm{Mn}_{x}\right)_{2} \mathrm{O}_{7}$ single crystals

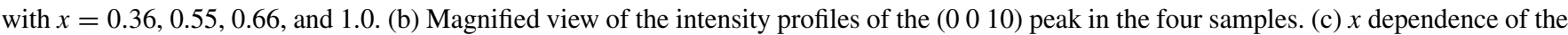
lattice parameter $c$ in $\mathrm{Sr}_{3}\left(\mathrm{Ru}_{1-x} \mathrm{Mn}_{x}\right)_{2} \mathrm{O}_{7}$. The error bars represent the standard errors. Note that the relative shift in the peak position observed in (b) and the decrease in lattice parameter $c$ observed in (c) agree with the difference in the effective ionic radius between Ru and Mn.

$\Delta$ increases from $\sim 0.05 \mathrm{eV}$ for $x=0.36$ to $\sim 0.15 \mathrm{eV}$ for $x=1.0$ [see the inset of Fig. 1(b)].

Figure 1(c) shows the temperature dependence of the magnetic susceptibility $\chi_{a b}$ measured under $H=1$ Tesla along the $a b$ plane for $x=0.36,0.55,0.66$, and 1 . Note that, for $x<1$, the magnitude of $\chi_{a b}$ increases with increasing $x$, indicating enhanced magnetic interactions with increasing the $\mathrm{Mn}$ content. For $0.36 \leqslant x \leqslant 0.66, \chi_{a b}$ increases with decreasing temperature until $T_{\mathrm{M}}$, indicated by black arrows in Fig. 1(c), below which the zero-field cooled (ZFC) susceptibility is highly suppressed, whereas the field cooled (FC) susceptibility only shows a slight kink. In contrast, $\chi_{a b}$ for $\mathrm{Sr}_{3} \mathrm{Mn}_{2} \mathrm{O}_{7}$ $(x=1)$ has a distinct characteristic, as highlighted in the inset of Fig. 1(c). Upon decreasing temperature, $\chi_{a b}$ initially increases until $\sim 300 \mathrm{~K}$, which then decreases. It increases again below $T_{\mathrm{M}} \sim 160 \mathrm{~K}$. This peculiar behavior is observed in both ZFC and FC curves.

Based on data shown above and previous work [1], we construct a complete phase diagram for $\mathrm{Sr}_{3}\left(\mathrm{Ru}_{1-x} \mathrm{Mn}_{x}\right)_{2} \mathrm{O}_{7}$, which is presented in Fig. 1(d). It consists of two phase boundary lines: one MIT line that separates the high-temperature metallic phase (Region I) from the low-temperature insulating phase and another magnetic transition line $\left(T_{\mathrm{M}}\right)$ that separates the high-temperature paramagnetic insulating phase (Region II) from the low-temperature magnetic phases. Region III represents the short-range AFM region in the low

TABLE I. Nominal $\mathrm{Mn} /(\mathrm{Mn}+\mathrm{Ru})$ ratio $(x)$ values in the four single-crystal samples investigated along with the $x$ values measured from EDS measurements.

\begin{tabular}{lcc}
\hline \hline Sample & $\begin{array}{c}\text { Nominal } x \text { value in } \\
\mathrm{Sr}_{3}\left(\mathrm{Ru}_{1-x} \mathrm{Mn}_{x}\right)_{2} \mathrm{O}_{7}\end{array}$ & $\begin{array}{c}\text { Measured } x \text { value } \\
\text { from EDS }\end{array}$ \\
\hline 1 & 0.3 & $0.36 \pm 0.01$ \\
2 & 0.6 & $0.55 \pm 0.02$ \\
3 & 0.75 & $0.66 \pm 0.02$ \\
4 & 1.0 & - \\
\hline \hline
\end{tabular}

Mn-doping region. The long-range AFM interactions are stabilized upon further increasing $x$, resulting in an E-type AFM order for $0.06<x<0.2[8,21]$ with the maximum transition temperature located at $x \sim 0.16$ (Region IV). The dome-shaped $T_{\mathrm{M}}$ vs $x$ indicates that there are competing magnetic interactions, which reduce $T_{\mathrm{M}}$ at $x>0.16$. Remarkably, $T_{\mathrm{M}}$ reaches the minimum at $x \sim 0.5$, i.e., $\mathrm{Ru}: \mathrm{Mn}=1: 1$ (Region $\mathrm{V}$ ). On the other end of the phase diagram, pure $\mathrm{Sr}_{3} \mathrm{Mn}_{2} \mathrm{O}_{7}(x=1)$ was reported to be a G-type AFM below the Néel temperature $T_{\mathrm{N}}=160 \mathrm{~K}$ [20]. Thus, it can be inferred that a long-range AFM ordering is again established in the system with high Mn concentration $(x>0.66$, Region VI).

To illuminate the nature of the magnetic ground states, we explore the magnetic properties of $x=0.36,0.55,0.66$, and 1.0 in detail. Figure 3(a) shows $\chi(T)$ for $x=0.55$ by applying $H=1 \mathrm{~T}$ along the $a b$ (red data) and $c$ (blue data) directions, respectively. Overall, the magnitude of $\chi_{a b}>\chi_{c}$, indicating magnetic anisotropy. The inset of Fig. 3(a) shows the temperature dependence of the inverse magnetic susceptibility $(1 / \chi)$ taken under both ZFC and FC conditions. The experimental data at $T \gg T_{\mathrm{M}}$ can be described well by the Curie-Weiss formula $\frac{1}{\chi-\chi_{0}}=\frac{T-\theta_{\mathrm{CW}}}{C}$, where $\chi_{0}$ is the temperature independent susceptibility, $\theta_{\mathrm{CW}}$ is the Curie-Weiss temperature, and $C$ is the Curie-Weiss constant. From the fit, represented by the solid lines in the inset of Fig. 3(a), we obtained $\chi_{0}^{a b}=$ $-1.03 \times 10^{-3} \mathrm{emu} / \mathrm{mol}-\mathrm{Oe}, C^{a b}=3.136(7) \mathrm{emu}-\mathrm{K} / \mathrm{mol}-\mathrm{Oe}$, and $\theta_{\mathrm{CW}}^{a b}=-28.4(3) \mathrm{K}$ for $H \| a b$ and $\chi_{0}^{c}=-1.05 \times$ $10^{-3} \mathrm{emu} / \mathrm{mol}-\mathrm{Oe}, C^{a b}=3.19(1) \mathrm{emu}-\mathrm{K} / \mathrm{mol}-\mathrm{Oe}$, and $\theta_{\mathrm{CW}}^{c}$ $=-40.8(8) \mathrm{K}$ for $H \| c$. The negative values of $\theta_{\mathrm{CW}}$ indicate that the magnetic coupling is dominantly AFM in both directions. Furthermore, from the fitted values of $C$, we can estimate the effective moment to be $\sim 5 \mu_{\mathrm{B}} / \mathrm{f}$.u.

Despite both the in-plane and out-of-plane magnetic interactions being seemingly AFM, the transition temperature $T_{\mathrm{M}}<\theta_{\mathrm{CW}}^{a b}$ and $\theta_{\mathrm{CW}}^{c}$ and the susceptibility curves do not represent trivial antiferromagnetism. To gain insight into magnetic interactions, we have measured the isothermal $M_{a b}(H)$ at different temperatures, as displayed in Fig. 3(b). Note that 
(a)

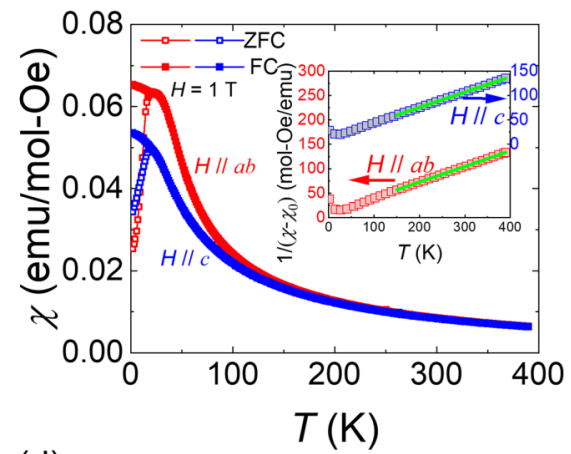

(d)

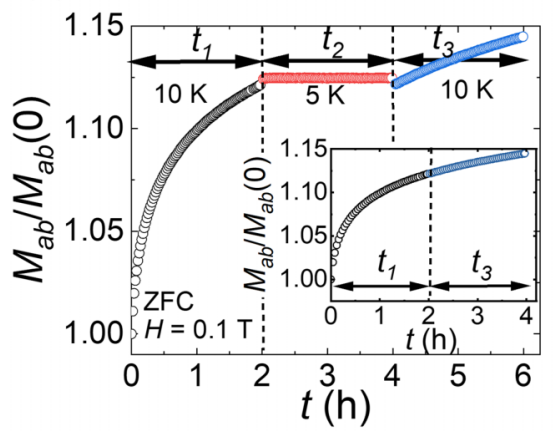

(b)

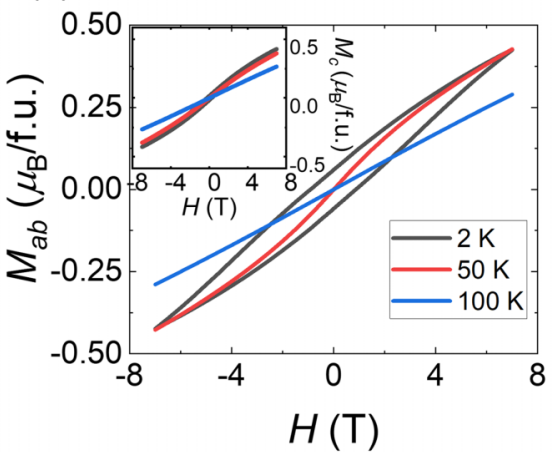

(e)

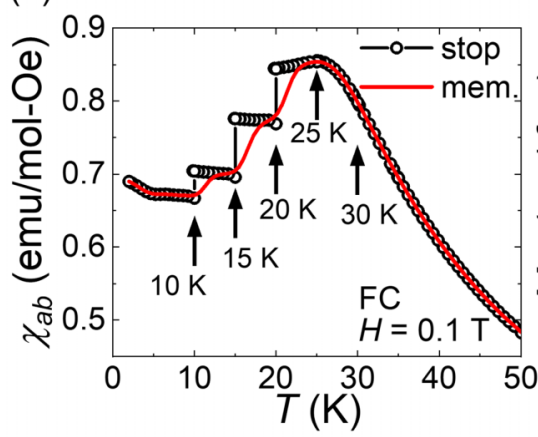

(c)

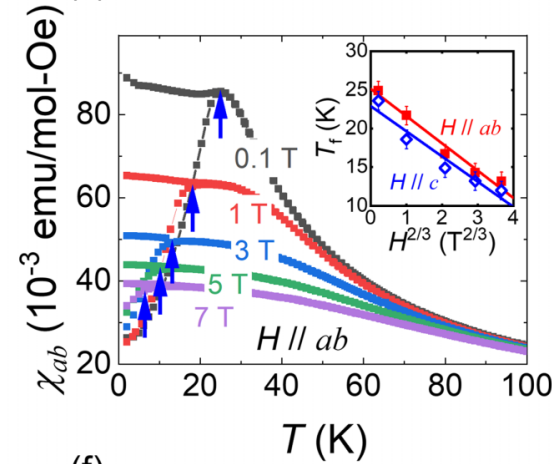

(f)

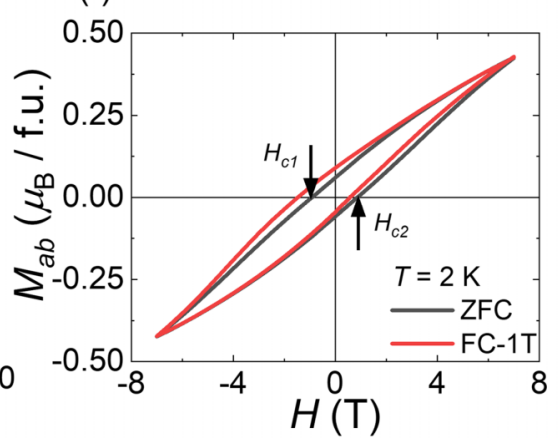

FIG. 3. Magnetic properties of $\mathrm{Sr}_{3}\left(\mathrm{Ru}_{1-x} \mathrm{Mn}_{x}\right)_{2} \mathrm{O}_{7}$ with $x=0.55$. (a) Temperature dependence of magnetic susceptibility $\chi$ measured under magnetic field $H=1 \mathrm{~T}$ parallel to the $a b$ (red) and $c$ (blue) directions. Both zero-field cooled (ZFC) and field cooled (FC) data are presented. Inset: Temperature dependence of the inverse susceptibilities in both directions and the respective Curie-Weiss fits (solid lines). (b) Field dependence of magnetization $M(H)$ at 2, 50, and $100 \mathrm{~K}$ for $H \| a b$ (main panel) and $c$ (inset) directions. (c) Temperature dependence of $\chi_{a b}$ under various indicated $H$. The arrows mark the freezing temperatures $T_{f}$. Inset: $T_{f}$ vs $H^{2 / 3}$ for both $H \| a b$ (red) and $H \| c$ (blue) configurations. The linear fits correspond to the de Almeida-Thouless relationship. (d) Time dependence of ZFC $M_{a b}$ under $H=0.1 \mathrm{~T}$ at $10 \mathrm{~K}$ with a temporary cooling to $5 \mathrm{~K}$. Inset: Data points from $t_{1}$ and $t_{3}$ plotted together highlighting magnetic memory. (e) Temperature dependence of FC $\chi_{a b}$ measured with stops at temperatures indicated by the arrows (black circles) and the memory (red) curve. (f) Comparison of $1 \mathrm{~T}$ FC $M_{a b}(H)$ curve with the ZFC $M_{a b}(H)$ curve at $2 \mathrm{~K}$.

$M_{a b}$ varies linearly with $H$ at $100 \mathrm{~K}$ but nonlinearly at 50 $\mathrm{K}$. Below $T_{\mathrm{M}}$, a large hysteretic $M(H)$ loop develops in both the $a b$ and $c$ directions below $T_{\mathrm{M}}$. This clearly indicates that, in addition to AFM interaction, there is FM interaction as well. The mixed AFM and FM interactions with unsaturated $M$ up to $7 \mathrm{~T}$ suggest the presence of the SG magnetic state. To confirm, we measure the magnetic susceptibility under a different field. As depicted in Fig. 3(c), both $T_{M}$ and the magnitude of $\chi$ decrease with increasing $H$. In canonical SGs, the freezing temperature $\left(T_{f}=T_{\mathrm{M}}\right)$ and $H$ follow the de Almeida-Thouless relationship [22,23] $H=\alpha\left(T_{\mathrm{SG}}-T_{f}\right)^{3 / 2}$, where $T_{\mathrm{SG}}$ is the SG transition temperature for $H=0$, and $\alpha$ is a function of magnetic interaction strengths. As shown in the inset of Fig. 3(c), the $T_{f}$ decreases monotonously with increasing $H$ with $T_{f} \sim H^{2 / 3}$ in both the $a b$ and $c$ directions, consistent with the SG behavior. From the linear fits, represented by the solid lines, we obtain $T_{\mathrm{SG}}^{a b} \sim 25 \mathrm{~K}$ and $a_{a b} \sim 19 \mathrm{~T}$ and $T_{\mathrm{SG}}^{a b} \sim 22 \mathrm{~K}$ and $\alpha_{c} \sim 18 \mathrm{~T}$.

An outstanding characteristic of SG is its nonequilibrium phenomena such as time-dependent magnetization, magnetic memory effect, and exchange bias (EB) [24-27]. Figure 3(d) shows the time dependence of ZFC $M_{a b}$ (see the Appendix for detail) performed at $T_{0}=10 \mathrm{~K}$ under $H=0.1 \mathrm{~T}$. At $T_{0}$, $M_{a b}$ evolves with time during $t_{1}=1 \mathrm{~h}$ following a stretched exponential function. Despite the sample being cooled to 5
$\mathrm{K}$ for $t_{2}=1 \mathrm{~h}$, the initial magnetic state is retained when it is warmed back to $T_{0}=10 \mathrm{~K}\left(t_{3}=1 \mathrm{~h}\right)$. As illustrated in the inset of Fig. 3(d), the data collected during $t_{1}$ and $t_{3}$ are not affected by the cooling history during the $t_{2}$ duration, demonstrating the magnetic memory effect. Further verification of the magnetic memory can be seen in the FC magnetization (see the Appendix for detail), shown in Fig. 3(f), where the data (black) were collected while cooling under $H=0.1 \mathrm{~T}$ with temporary stops of $\Delta t=1 \mathrm{~h}$ with the removal of the applied field at temperatures indicated by black arrows. Once $\Delta t=1 \mathrm{~h}$ had elapsed, the field was reapplied, and cooling resumed. After reaching $2 \mathrm{~K}$, the memory curve [red curve in Fig. 3(d)] was measured without any stops in the warming mode. Clearly, the system was "aged" by stopping during the cooling cycle, confirming the magnetic memory effect.

In addition to the magnetic memory effect, we also observed a prominent $\mathrm{EB}$ effect below $T_{\mathrm{SG}}$, which is characterized by a shift in the magnetization hysteresis curve [28,29]. Figure 3(f) shows the $M_{a b}(H)$ curve measured at $T=2 \mathrm{~K}$ under both the ZFC (black curve) and FC (red) conditions. A leftward shift can be clearly seen in the FC curve compared with the ZFC curve. The exchange field $H_{E}$ is quantified as $H_{E}=\left(H_{c 1}+H_{c 2}\right) / 2$, where $H_{c 1}$ and $H_{c 2}$ are the left and right coercive fields in the FC curve [indicated in the Fig. 3(f) by arrows], respectively. From the data taken under $1 \mathrm{~T}$ field 
(a)

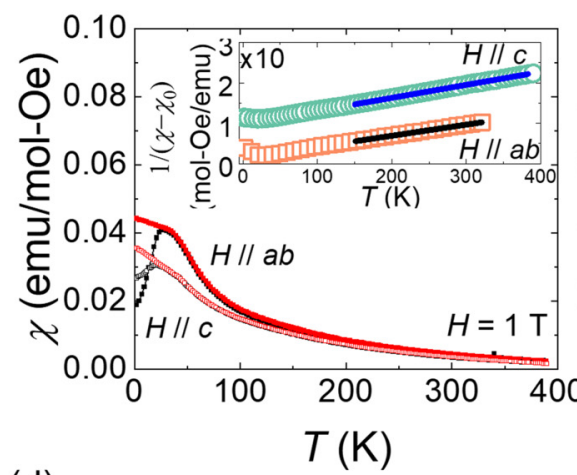

(d)

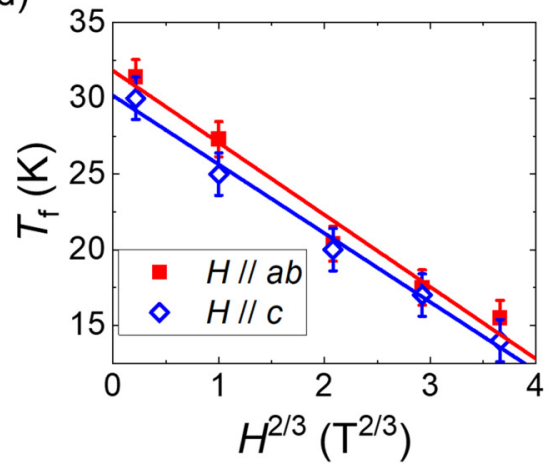

(b)

$$
x=0.36
$$

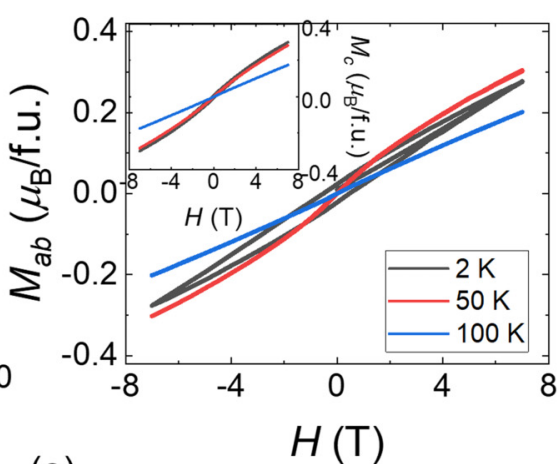

(e)

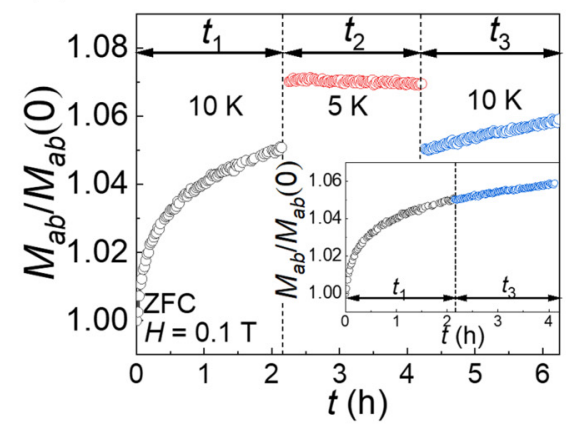

(c)

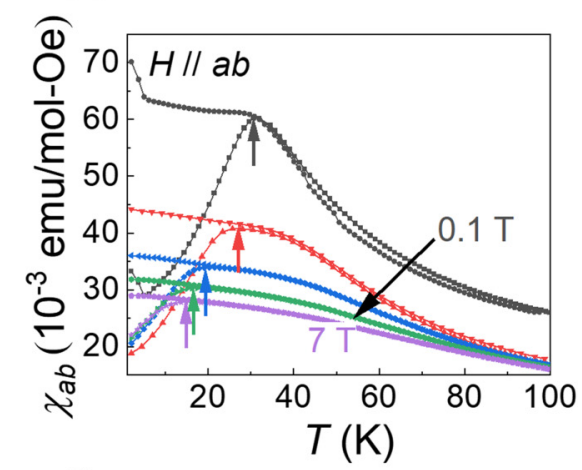

(f)

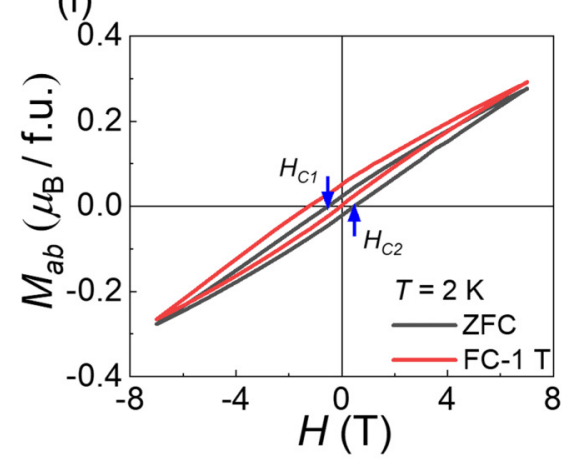

FIG. 4. Magnetic properties of $\mathrm{Sr}_{3}\left(\mathrm{Ru}_{1-x} \mathrm{Mn}_{x}\right)_{2} \mathrm{O}_{7}$ with $x=0.36$. (a) Temperature dependence of $\chi$ with $H=1 \mathrm{~T}$ parallel to $a b$ and $c$ directions, respectively. Inset: Temperature dependence of inverse susceptibility shifted vertically for clarity and Curie-Weiss fitting (solid lines). (b) Field dependence of isothermal magnetization at various temperatures with $H \| a b$ (main panel) and $H \| c$ (inset). (c) $\chi_{a b}(T$ ) curves at indicated temperatures with arrows for $T_{f}(H)$. (d) $T_{f}$ as a function of $H^{2 / 3}$ for $H \| a b$ and $H \| c$ with fits represented by solid lines. (e) Magnetic relaxation at $10 \mathrm{~K}$ for time durations $t_{1}$ and $t_{3}$ with a temporary cooling to $5 \mathrm{~K}$ for time duration $t_{2}$. Inset: Data at $10 \mathrm{~K}$ during $t_{1}$ and $t_{3}$ showing the magnetic memory effect. (f) ZFC $M_{a b}(H)$ curve at $2 \mathrm{~K}$ along with FC $M_{a b}(H)$ curve measured after cooling the sample to $2 \mathrm{~K}$ under $H=1 \mathrm{~T}$.

cooling, we obtain $H_{E} \sim-0.45$ and -0.29 T for $H \| a b$ and $H \| c$, respectively. The presence of the EB below $T_{\mathrm{SG}}$ demonstrates the existence of disparate magnetic orders [28,29].

The SG ground state was observed in the $0.36 \leqslant x \leqslant$ 0.66 region of the phase diagram (see Figs. 4 and 5). The emergence of $\mathrm{SG}$ in $\mathrm{Sr}_{3}\left(\mathrm{Ru}_{1-x} \mathrm{Mn}_{x}\right)_{2} \mathrm{O}_{7}$ is reminiscent of the cluster-SG state in $\left(\mathrm{Sr}_{1-x} \mathrm{Ca}_{x}\right)_{3} \mathrm{Ru}_{2} \mathrm{O}_{7}$ for $0.08<x<0.4$ [16]. This is attributed to the variation of the electronic structure caused by the structural distortion, resulting in the enhancement of FM correlation over the AFM fluctuations [16]. In the case of Mn-doped $\mathrm{Sr}_{3}\left(\mathrm{Ru}_{1-x} \mathrm{Mn}_{x}\right)_{2} \mathrm{O}_{7}$, the local structure is also altered by smaller Mn ions [5,30], namely, reduced octahedral distortion [1,5,31]. The puzzling fact is that $T_{\mathrm{SG}}$ reaches the minimum when $\mathrm{Ru} / \mathrm{Mn} \sim 1$ (i.e., $x \sim 0.5$ ), implying the SG state is associated with the interplay between $\mathrm{Ru}$ and $\mathrm{Mn}$ moments.

To understand the origin of the SG state, we examine the evolution of the magnetic properties with $x$. Figure 6(a) shows the $x$ dependence of the magnetic susceptibility anisotropy $\left(\chi_{a b} / \chi_{c}\right)$ at $T=300 \mathrm{~K}\left(>T_{\mathrm{SG}}\right)$. Though $\chi_{a b}>\chi_{c}$ for $x=0.36$, the anisotropy nearly vanishes for $x=0.55$ with $\chi_{a b} / \chi_{c} \sim 1$. Increasing $x$ further restores the magnetic anisotropy, reaching the maximum for $x=1.0\left(\chi_{a b} / \chi_{c}=\right.$ 1.8). Figure 6(b) shows the variation of the effective magnetic moment $\left(\mu_{\text {eff }}\right)$ obtained from Curie-Weiss fitting of the magnetic susceptibility data (see Figs. 4 and 5) with $x$, where a similar behavior is observed: $\mu_{\text {eff }}$ is approximately the same obtained from both $\chi_{a b}$ and $\chi_{c}$ and reaches the minimum for $x=0.55$. It should also be noted that, while there is a slight directional variation in $\mu_{\text {eff }}$ in $x=0.36$ and 0.66 , it is approximately isotropic for $x=0.55$. The isotropic behavior of $\mu_{\text {eff }}$ with the lowest $T_{\mathrm{SG}}$ for $x \sim 0.5$ signals the importance of intermixing of Ru and $\mathrm{Mn}$.

The random occupation of $\mathrm{Ru}$ and $\mathrm{Mn}$ can lead to complex magnetic interactions. A preliminary understanding can be achieved from the Curie-Weiss temperature $\theta_{\mathrm{CW}}$. As shown in Fig. 6(c), both $\theta_{\mathrm{CW}}^{a b}$ and $\theta_{\mathrm{CW}}^{c}$ are negative for $x=0.36$, indicating AFM interaction in both directions. Upon increasing $x$, both $\theta_{\mathrm{CW}}^{a b}$ and $\theta_{\mathrm{CW}}^{c}$ gradually change toward positive values. The apparent sign change of $\theta_{\mathrm{CW}}^{a b}$ indicates that there are both $\mathrm{AFM}$ and FM interactions with dominant FM interaction at $x \sim 0.66$. The mixed AFM and FM interactions are crucial in the establishment of the SG state. Therefore, it is important to determine the dopant distribution.

Figure 7(a) shows the high-angle annular dark-field (HAADF) STEM image along the [100] zone axis for $x=$ 0.16 . As the intensity of the imaged atoms is proportional to the atomic number, the bright spots should correspond to $\mathrm{Mn}, \mathrm{Ru}$, and $\mathrm{Sr}$. Indeed, we find that the atomic arrangement is consistent with that shown in Fig. 1(a). In the projected structure shown in Fig. 7(a), white dots represent $\mathrm{Sr}$, and green dots denote $\mathrm{Ru} / \mathrm{Mn}$. To detect the $\mathrm{Ru}$ and $\mathrm{Mn}$ distribution, 
(a)

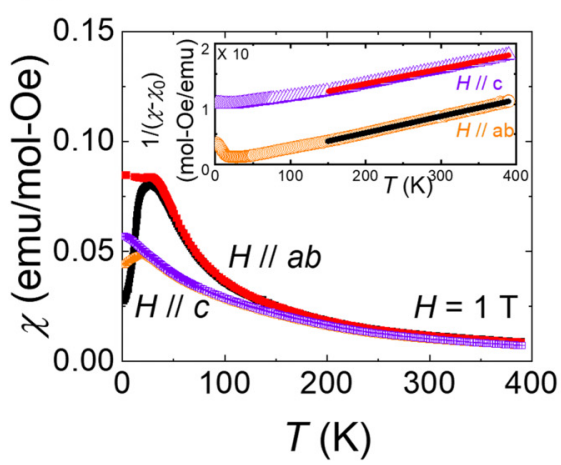

(d)

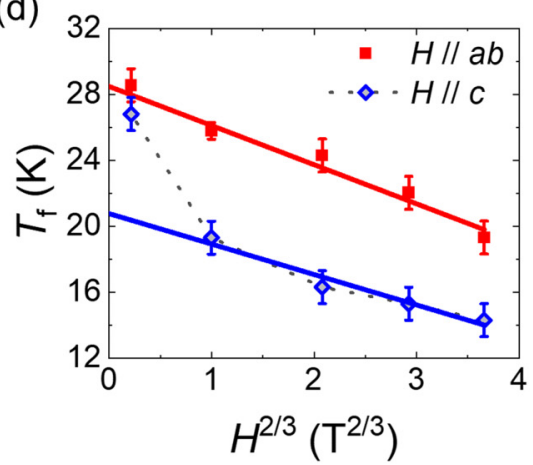

(b)

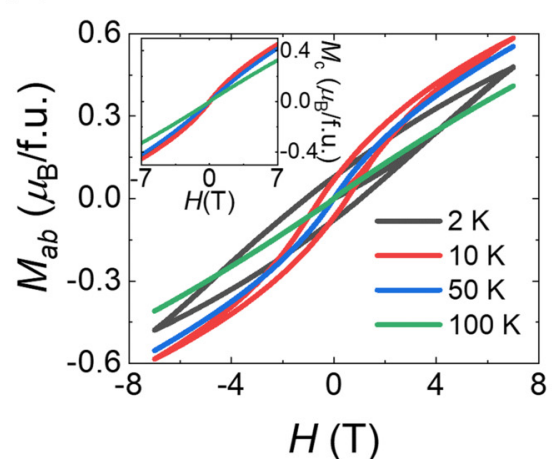

(e)

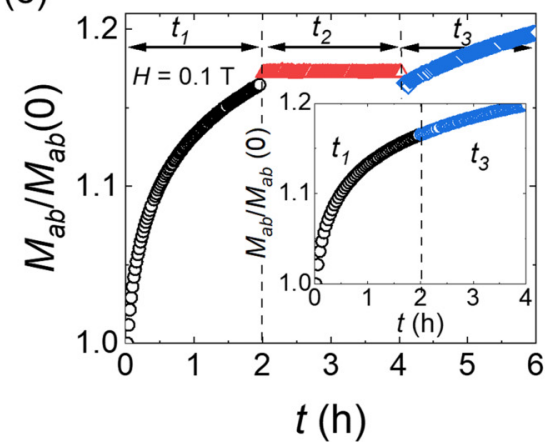

(c)

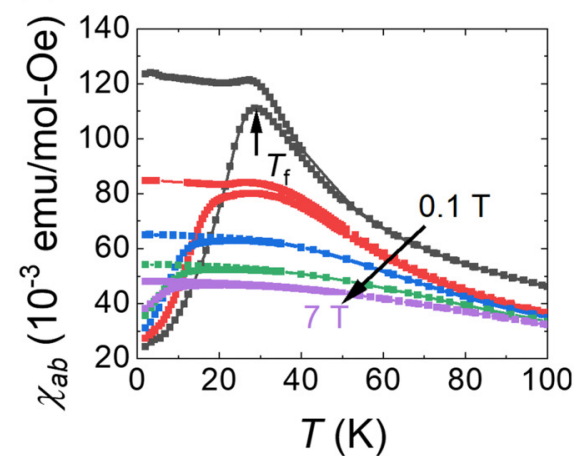

(f)

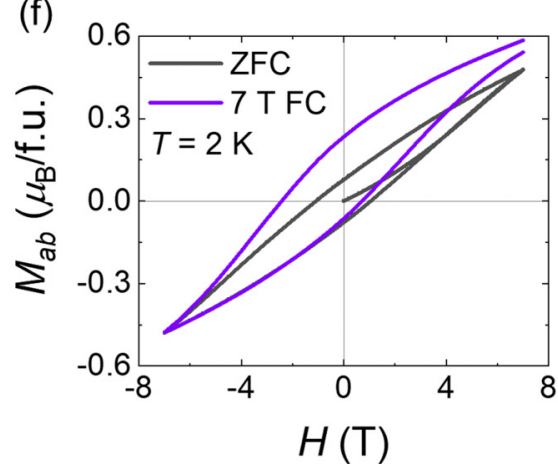

FIG. 5. Magnetic properties of $\mathrm{Sr}_{3}\left(\mathrm{Ru}_{1-x} \mathrm{Mn}_{x}\right)_{2} \mathrm{O}_{7}$ with $x=0.66$. (a) Temperature dependence of $\chi$ with $H=1 \mathrm{~T}$ parallel to the $a b$ and $c$ directions. Inset: Temperature dependence of inverse susceptibility shifted vertically for clarity and Curie-Weiss fitting (solid lines). (b) Field dependence of the isothermal magnetization at various temperatures with $H \| a b$ (main panel) and $H \| c$ (left inset). (c) $\chi_{a b}(T)$ curves with various fields. (d) $T_{f}$ as a function of $H^{2 / 3}$ for $H \| a b$ and $H \| c$ with fits represented by solid lines. (e) Magnetic relaxation at $15 \mathrm{~K}$ for time durations $t_{1}$ and $t_{3}$ with a temporary cooling to $10 \mathrm{~K}$ for time duration $t_{2}$. Inset: Data at $15 \mathrm{~K}$ during $t_{1}$ and $t_{3}$ showing the magnetic memory effect. (f) $\mathrm{ZFC} M_{a b}(H)$ curve at $2 \mathrm{~K}$ along with FC $M_{a b}(H)$ curve measured after cooling the sample to $2 \mathrm{~K}$ under $H=7 \mathrm{~T}$.

EDS measurement was carried out to map Ru and Mn along the [100] zone axis for $x=0.16$. Figure 7(b) shows the distribution of $\mathrm{Ru}$ (orange) and $\mathrm{Mn}$ (green), revealing randomness of Mn occupation in the Ru site (also see Fig. 8).

Due to a random distribution of $\mathrm{Mn}$, competing magnetic interactions between $\mathrm{Ru}$ and $\mathrm{Mn}$ moments lead to a sharp decrease in $T_{\mathrm{N}}$ above $x=0.16$. Further increase of $x$ apparently introduces stronger spin disorder. Figure 7(c) illustrates such a trend with competing FM and AFM interactions. At the maximum mixing (i.e., $\mathrm{Ru}: \mathrm{Mn}=1: 1$ ), the strongest $\mathrm{FM}$ and AFM competition results in the SG state with the low- est $T_{\mathrm{SG}}$ [27]. As illustrated in Fig. 7(d), the local magnetic landscape is comprised of segregated magnetic regions with competing short-range ordering that prevents the formation of a long-range magnetic order. This resembles the electronic phase separation near $T_{\text {MIT }}$ in $\mathrm{Sr}_{3}\left(\mathrm{Ru}_{1-x} \mathrm{Mn}_{x}\right)_{2} \mathrm{O}_{7}(x<0.1)$ as well as $\mathrm{Sr}_{3}\left(\operatorname{Ir}_{1-x} \mathrm{Ru}_{x}\right)_{2} \mathrm{O}_{7}$ [5,19]. While the MIT occurs at temperatures much higher than $T_{\mathrm{SG}}$ for $x \geqslant 0.36$, the presence of a sizable EB effect observed at $T<T_{\mathrm{SG}}$ substantiates the magnetic phase segregation scenario.

As mentioned above, the magnetic behavior of pure $\mathrm{Sr}_{3} \mathrm{Mn}_{2} \mathrm{O}_{7}(x=1)$ is different from that in the region of
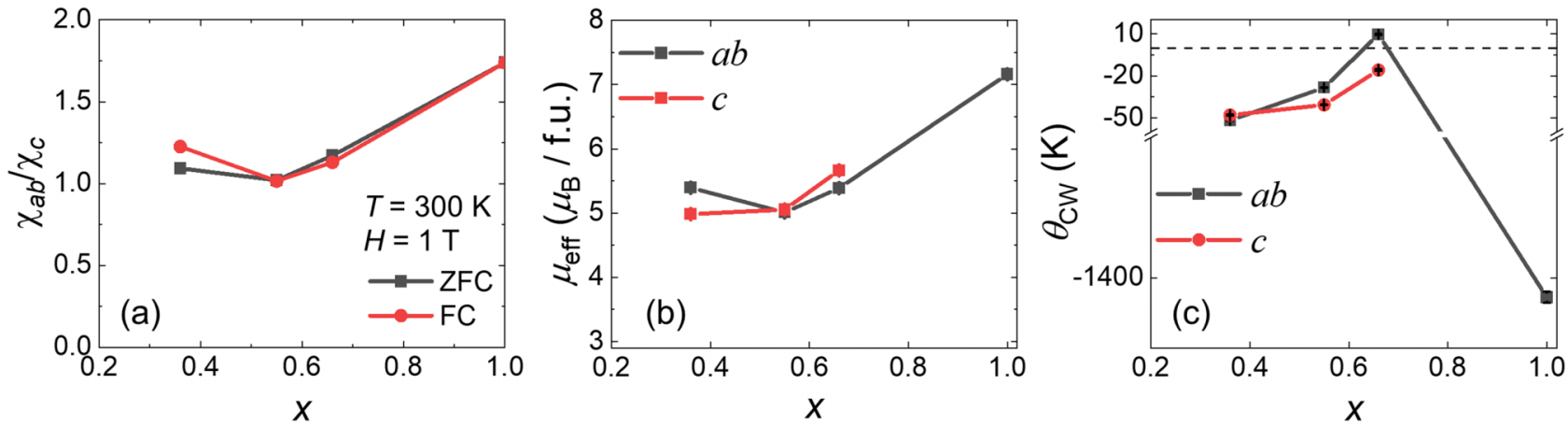

FIG. 6. Doping dependence of (a) $\chi_{a b} / \chi_{c}$ at $T=300 \mathrm{~K}$ with $H=1 \mathrm{~T}$, (b) effective magnetic moment per formula unit, and (c) Curie-Weiss temperature. The dashed line in (c) delineates the zero value of $\theta_{\mathrm{CW}}$. 

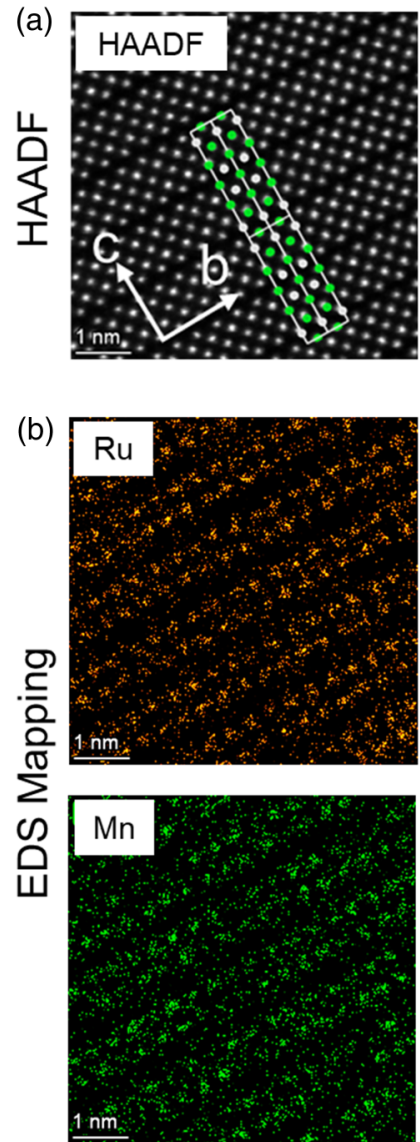

STEM (c)
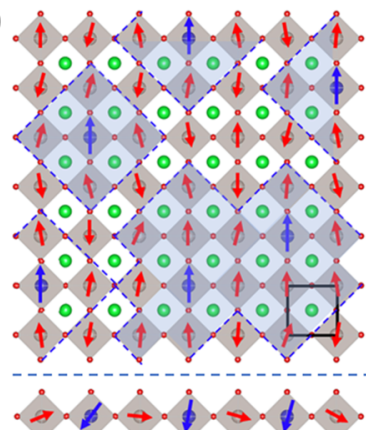

0.000 .00 .00

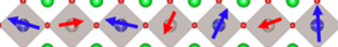

$\rightarrow 0000 \div 0 \div 0$.

$0 \div 0=0.000$

0000000.0

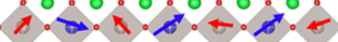

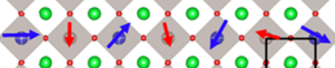

$\therefore: 1.60 .0 .0$
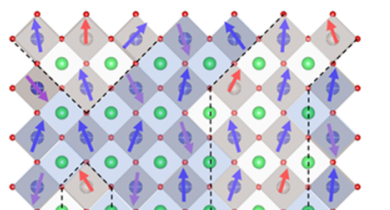

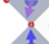

.

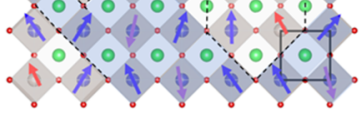

- $\bigcirc \bigcirc \mathrm{Sr} \odot \mathrm{Ru} \odot \mathrm{Mn}$ (d)
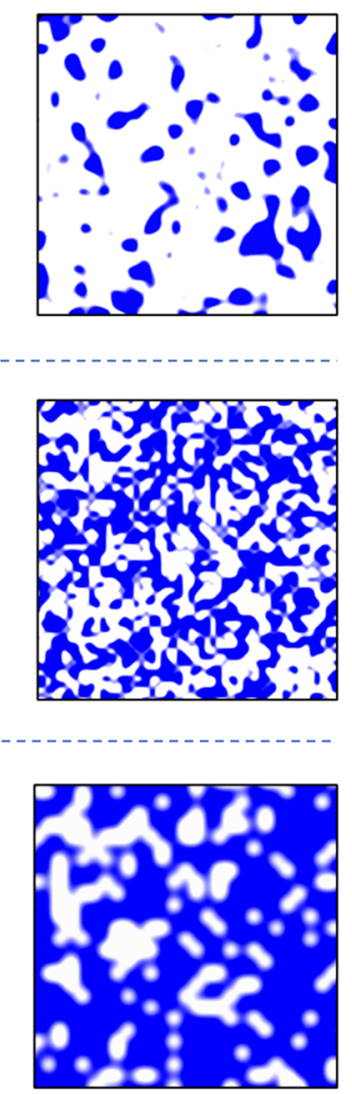

FIG. 7. (a) High-angle annular dark-field (HAADF) scanning transmission electron microscopy (STEM) image and (b) energy dispersive $\mathrm{x}$-ray spectroscopy (EDS) elemental mapping of $\mathrm{Ru}$ and $\mathrm{Mn}$ in $\mathrm{Sr}_{3}\left(\mathrm{Ru}_{1-x} \mathrm{Mn}_{x}\right)_{2} \mathrm{O}_{7}$ with $x=0.16$ taken along the [100] zone axis. Simulated crystal structure is overlaid in (a). (c) Visualization of the local spin randomness within the $(\mathrm{Ru} / \mathrm{Mn}) \mathrm{O}_{6}$ plane. Arrows represent magnetic moments of $\mathrm{Mn}$ (blue) and $\mathrm{Ru}$ (red). The short-range correlation between the $\mathrm{Mn}$ and $\mathrm{Ru}$ spins creates spin clusters, represented by the shaded region, giving rise to the spin-glass (SG) state. (d) Depiction of the evolution of competing magnetic regions (blue and white areas) in $(\mathrm{Ru} / \mathrm{Mn}) \mathrm{O}_{6}$ planes upon increasing $x$.

$0.36 \leqslant x \leqslant 0.66$. Figure 9(a) shows the temperature dependence of $\chi_{a b}$ and $\chi_{c}$ for $\mathrm{Sr}_{3} \mathrm{Mn}_{2} \mathrm{O}_{7}$ between 2 and $800 \mathrm{~K}$. Note that both $\chi_{a b}$ and $\chi_{c}$ show prominent kinks at $\sim 160 \mathrm{~K}$. While the temperature at which the anomaly occurs agrees with the reported AFM transition at $T_{\mathrm{N}}=160 \mathrm{~K}$ [20], its nature is atypical: there is a broad hump centered $\sim 2 T_{\mathrm{N}}$. This indicates the occurrence of strong spin fluctuations at $T$ much greater than $T_{\mathrm{N}}$. Likewise, the inverse susceptibility does not (a)

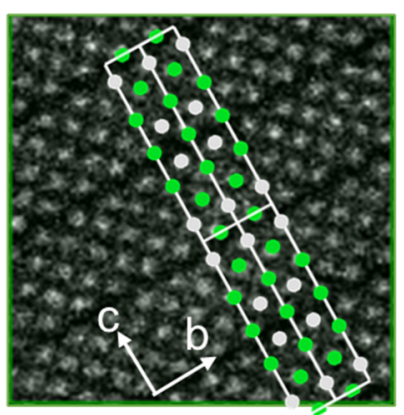

HAADF (b)

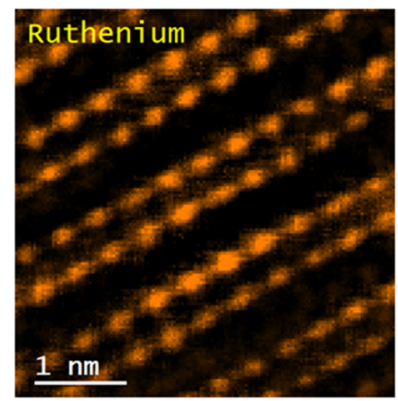

(c)

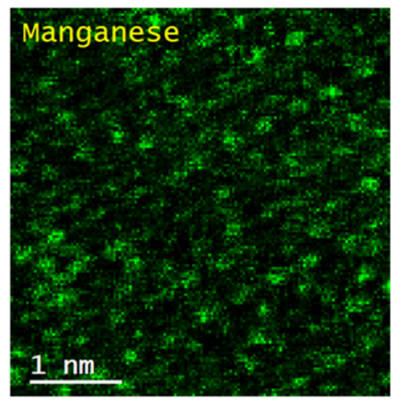

EELS Mapping

FIG. 8. (a) High-angle annular dark-field (HAADF) scanning transmission electron microscopy (STEM) image and electron energy loss spectroscopy (EELS) elemental mapping of (b) $\mathrm{Ru}$ and (c) $\mathrm{Mn}$ in $\mathrm{Sr}_{3}\left(\mathrm{Ru}_{1-x} \mathrm{Mn}_{x}\right)_{2} \mathrm{O}_{7}$ with $x=0.16$ taken along the [100] zone axis. Simulated crystal structure is overlaid in (a). 

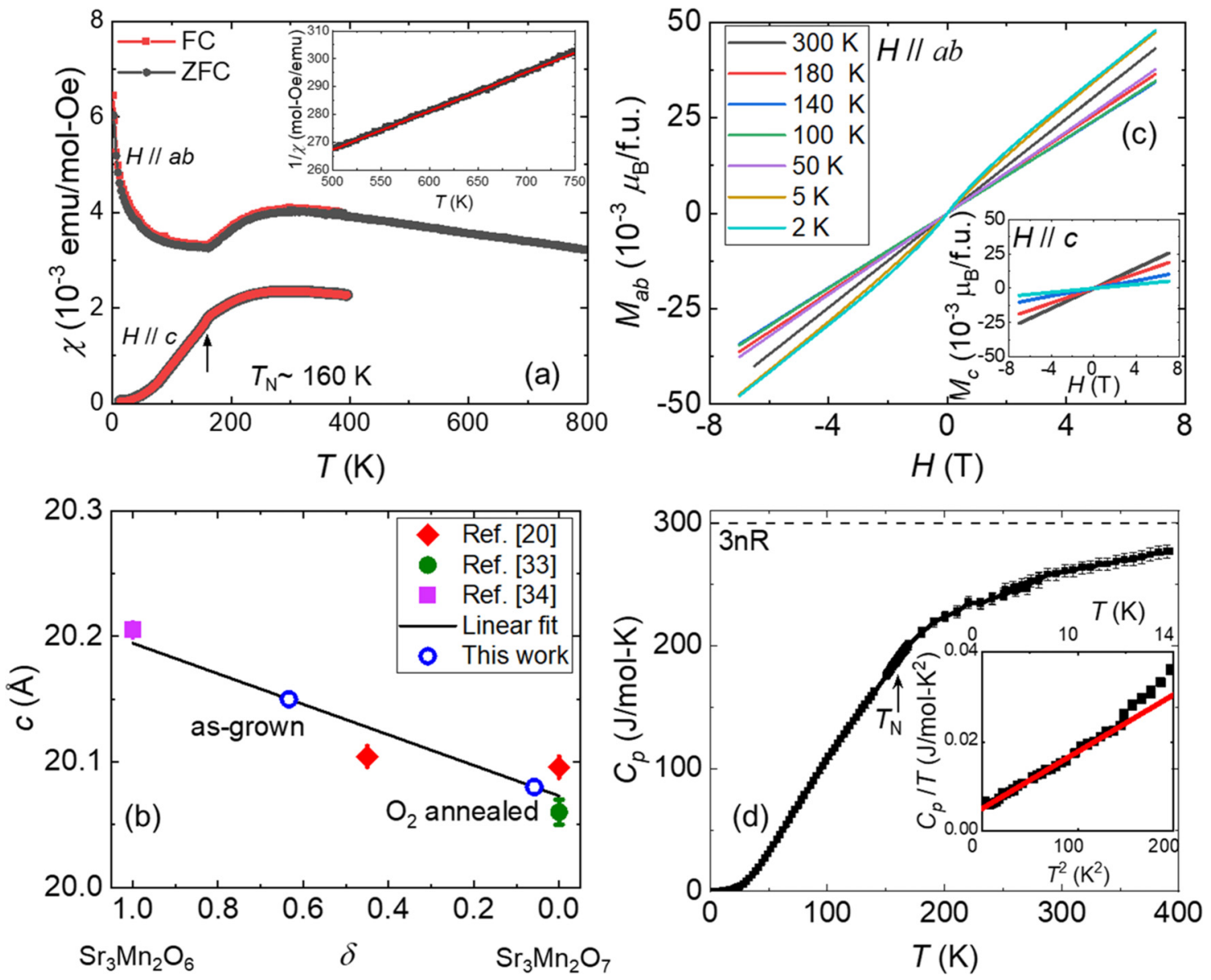

FIG. 9. (a) Temperature dependence of magnetic susceptibility in $\mathrm{Sr}_{3} \mathrm{Mn}_{2} \mathrm{O}_{7}$ measured under $H=1 \mathrm{~T}$ along the $a b$ and $c$ directions. (b) Relationship between oxygen deficiency $\delta$ and lattice parameter $c$ in $\mathrm{Sr}_{3} \mathrm{Mn}_{2} \mathrm{O}_{7-\delta}$. The solid points are from Refs. [20,33,34], the solid line represents a linear fit, and the open circles are from this paper. (c) Field dependences of isothermal magnetization with $H \| a b$ (main panel) and $H \| c$ (inset) measured at indicated temperatures. (d) Temperature dependence of specific heat $C_{p}$. Inset: Low-temperature behavior plotted as $C_{p} / T$ vs $T^{2}$. Solid line represents linear fit.

conform to the Curie-Weiss law until $\sim 500 \mathrm{~K}$. The inset of Fig. 9(a) demonstrates the excellent fit of the experimental data by the Curie-Weiss law with $\mu_{\text {eff }} \sim 7.56 \mu_{\mathrm{B}} /$ f.u. and $\theta_{\mathrm{CW}}=-1413 \mathrm{~K}$, which are also plotted in Figs. 6(b) and 6(c). Compared with $T_{N}, \theta_{\mathrm{CW}}$ is much larger. This may be related to the layered structure, as discussed in Ref. [32]. The obtained $\mu_{\text {eff }}$ is also higher than that for $\mathrm{Mn}^{4+}$ with maximum $S_{\mathrm{Mn} 4+}=\frac{3}{2}$ [20,32]. Our $\mu_{\text {eff }}$ requires that the total spin $S_{\mathrm{Mn}} \sim 2$, which corresponds to $\mathrm{Mn}^{3+}$. If this is the case, oxygen deficiency $\delta$ is expected with chemical composition $\mathrm{Sr}_{3} \mathrm{Mn}_{2} \mathrm{O}_{7-\delta}$. Figure 9(b) shows the $\delta$ dependence of the lattice parameter $c$ in $\mathrm{Sr}_{3} \mathrm{Mn}_{2} \mathrm{O}_{7-\delta}$ obtained from the reports in Refs. $[20,33,34]$, where a linear trend can be observed. The lattice parameter $c$ of both as-grown crystal $[c=20.15(2) \AA]$ and $\mathrm{O}_{2}$ annealed crystal $[c=20.08(2) \AA]$ used in this paper fit well with this relationship. From the linear fit, we estimate our as-grown and $\mathrm{O}_{2}$ annealed crystals to have the oxygen deficiency $\delta_{\text {as-grown }} \sim 0.64$ and $\delta_{\mathrm{O} 2 \text {-annealed }} \sim 0.06$, respectively. The prolonged annealing in $\mathrm{O}_{2}$ pushes the crystal close to stoichiometry, resulting in the same AFM transition temperature as that for $\mathrm{Sr}_{3} \mathrm{Mn}_{2} \mathrm{O}_{7}$ [14]. Based on this, the high effective moment cannot be attributed to $\mathrm{Mn}^{3+}$ but rather the larger $g$ factor $(>2)$.
At present, the magnetic properties of $\mathrm{Sr}_{3} \mathrm{Mn}_{2} \mathrm{O}_{7-\delta}$ remain unsettled. According to Ref. [20], $\mathrm{Sr}_{3} \mathrm{Mn}_{2} \mathrm{O}_{7}$ forms a G-type AFM ordering $<160 \mathrm{~K}$ based on neutron scattering investigation on the polycrystalline sample. In our case, while $\chi_{c}$ deceases, $\chi_{a b}$ is enhanced below $T_{\mathrm{N}}=160 \mathrm{~K}$ [see Fig. 9(a)]. This indicates the prominent FM interaction in the $a b$ plane, thus pointing toward a canted spin configuration where the spins are coupled AFM in the $c$ direction but FM in the $a b$ plane. This assertion is supported by the field dependence of $M$ shown in Fig. 9(c), where a nonlinear behavior is developed at low temperatures for $M_{a b}$ while $M_{c}$ depends linearly on $H$ (see the inset of Fig. 9(c)). Such information may be difficult to probe in polycrystalline samples, which is the averaged result in all directions. On the other hand, a canted AFM ordering $(<\sim 250 \mathrm{~K})$ has been observed for $\mathrm{Sr}_{3} \mathrm{Mn}_{2} \mathrm{O}_{6}$ [34]. We find that the as-grown crystal $\left(\mathrm{Sr}_{3} \mathrm{Mn}_{2} \mathrm{O}_{6.36}\right)$ does not show any sign of magnetic ordering down to $2 \mathrm{~K}$ (see Fig. 10), agreeing with that for $\mathrm{Sr}_{3} \mathrm{Mn}_{2} \mathrm{O}_{6.55}$ $(\delta=0.45)[20]$.

A typical magnetic transition would manifest its signatures in thermodynamic properties as excess entropy is released when spins form a long-range order. To gain further insight into the nature of the transition at $T_{\mathrm{N}}$ in our nearly 


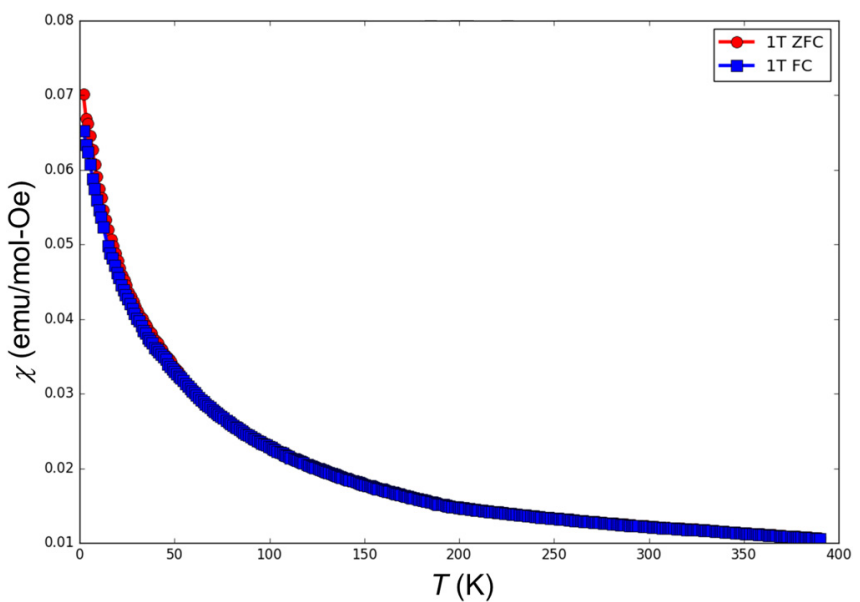

FIG. 10. Temperature dependence of the magnetic susceptibility in as-grown $\mathrm{Sr}_{3} \mathrm{Mn}_{2} \mathrm{O}_{7-\delta}$ single crystal measured under $H=1 \mathrm{~T}$.

stoichiometric $\mathrm{Sr}_{3} \mathrm{Mn}_{2} \mathrm{O}_{7}$ crystals, we measure its specific heat $C_{p}$. Figure 9(d) shows the temperature dependence of $C_{p}$ for $\mathrm{Sr}_{3} \mathrm{Mn}_{2} \mathrm{O}_{6.94}$ under the zero field, demonstrating a smooth variation with temperature without noticeable anomaly near $T_{N}$. This suggests that most of the magnetic entropy is released at higher temperatures before $T_{\mathrm{N}}$, in agreement with the presence of broad humps in the magnetic susceptibility at a temperature well above $T_{\mathrm{N}}$. In an AFM system, $C_{p}=$ $C_{\text {phonon }}+C_{\mathrm{AFM}}$, where $C_{\text {phonon }}=\beta T^{3}$ is the phonon specific heat, and $C_{\mathrm{AFM}}$ varies exponentially with $T$, which is related to the spin gap [35]. The inset of Fig. 9(d) shows $C_{p} / T$ as a function of $T^{2}$, with linear behavior $<\sim 12 \mathrm{~K}$. This implies that $C_{\mathrm{AFM}}$ is negligible at low temperatures. While the slope corresponds to $\beta=0.127 \mathrm{~mJ} / \mathrm{mol}-\mathrm{K}^{4}$, we also observe a nonzero constant $\left(\sim 5 \mathrm{~mJ} / \mathrm{mol}^{-\mathrm{K}^{2}}\right)$, indicating the presence of a linear term in the specific heat. Given an insulating system, a linear contribution should not be attributed to the electronic specific heat. While several scenarios are proposed [36], most relevant to our system is the existence of a magnetic amorphous phase, as suggested for another manganite [37], and/or contributions from oxygen deficiency [38].

\section{CONCLUSIONS}

In summary, through the characterization of high-quality single crystals of $\mathrm{Sr}_{3}\left(\mathrm{Ru}_{1-x} \mathrm{Mn}_{x}\right)_{2} \mathrm{O}_{7}$ with $0.36 \leqslant x \leqslant 1.0$, we have demonstrated the intricate FM and AFM interplay between the spins and untangled the peculiar evolution of the magnetic ground state in the series. The intermixing of $\mathrm{Mn}$ and $\mathrm{Ru}$ leads to spin disorder due to the complexities of the magnetic interactions. Specifically, the SG ground state is observed in the region of $0.36 \leqslant x \leqslant 0.66$. At $x \sim 0.5$ (i.e., $\mathrm{Ru}: \mathrm{Mn}=1: 1)$, the system exhibits the lowest SG transition temperature, implying the strongest competition between AFM and FM interactions. The Ru and Mn intermixing provides the necessary components for the local magnetic phase segregation leading to the SG with a large EB. As $x$ approaches 1, the in-plane FM and $c$-direction AFM become less combative but more cooperative. The system thus forms a long-range AFM order with antiparallel spins along the $c$ direction but FM in the $a b$ plane, i.e., canted AFM. Our experimental observations also demonstrate that the tuning magnetic interactions and imposing randomness is a viable path to generate exotic spin states.

\section{ACKNOWLEDGMENTS}

This work described in this paper was initially supported by the U.S. Department of Energy under EPSCoR Grant No. DESC0016315. However, the work was completed through additional support by the U.S. National Science Foundation under Grant No. DMR-1504226. We thank the Shared Instrumentation Facility at Louisiana State University for facilitating the XRD and EDS measurements.

\section{APPENDIX: PROCEDURES FOR MEMORY EFFECT EXPERIMENTS}

For the ZFC measurement, the sample was cooled from $T \gg T_{\mathrm{SG}}$ under $H=0$. After reaching $T_{0}=10 \mathrm{~K}, H=0.1 \mathrm{~T}$ was applied, and $M$ was measured as a function of time $t$ for a time duration $t_{1}=2 \mathrm{~h}$. After $t_{1}$, the sample was cooled rapidly to $5 \mathrm{~K}$, and $M$ was measured as a function of $t$ for another time duration $t_{2}=2 \mathrm{~h}$. After $t_{2}$ had elapsed, the sample was warmed back up to $T_{0}=10 \mathrm{~K}$, and $M$ was measured for time duration $t_{3}=2 \mathrm{~h}$.

For the FC measurement, the sample was cooled from $T \gg T_{\mathrm{SG}}$ under $H=0.1 \mathrm{~T}$ to the base temperature of $2 \mathrm{~K}$, and $M$ was measured as a function of $T$. During the cooling cycle, temporary stops were made at temperatures $T_{\text {stop }}=30$, $25,20,15$, and $10 \mathrm{~K}$. During each stop, the magnetic field was removed, and the sample was allowed to "age" by keeping it at that temperature for $t_{\text {stop }}=2 \mathrm{~h}$. After $t_{\text {stop }}$ had elapsed, the magnetic field was turned back on, and cooling was resumed. This temporary stop-and-aging process was performed at each $T_{\text {stop }}$ until the base temperature of $2 \mathrm{~K}$ was reached. The thusobtained $M(T)$ curve is termed the "stop" curve in this paper and Fig. 3(e). After reaching $2 \mathrm{~K}$, the sample was warmed up to $T \gg T_{\mathrm{SG}}$, and $M(T)$ was measured while the field was still on continuously without any stops. The data obtained in the warming cycle demonstrate the memory effect and are termed "mem." in this paper and Fig. 3(e).
[1] B. Hu, G. T. McCandless, V. O. Garlea, S. Stadler, Y. Xiong, J. Y. Chan, E. W. Plummer, and R. Jin, Structure-property coupling in $\mathrm{Sr}_{3}\left(\mathrm{Ru}_{1-x} \mathrm{Mn}_{x}\right)_{2} \mathrm{O}_{7}$, Phys. Rev. B 84, 174411 (2011).

[2] Y. Maeno, H. Hashimoto, K. Yoshida, S. Nishizaki, T. Fujita, J. G. Bednorz, and F. Lichtenberg, Superconductivity in a layered perovskite without copper, Nature (London) 372, 532 (1994).
[3] R. S. Perry, L. M. Galvin, S. A. Grigera, L. Capogna, A. J. Schofield, A. P. Mackenzie, M. Chiao, S. R. Julian, S. Nakatsuji, C. Pfleiderer, S. I. Ikeda, and Y. Maeno, Metamagnetism and Critical Fluctuations in High Quality Single Crystals of the Bilayer Ruthenate $\mathrm{Sr}_{3} \mathrm{Ru}_{2} \mathrm{O}_{7}$, Phys. Rev. Lett. 86, 2661 (2001).

[4] K. Kitagawa, K. Ishida, R. S. Perry, T. Tayama, T. Sakakibara, and Y. Maeno, Metamagnetic Quantum Criticality Revealed by 
${ }^{17} \mathrm{O}-\mathrm{NMR}$ in the Itinerant Metamagnet $\mathrm{Sr}_{3} \mathrm{Ru}_{2} \mathrm{O}_{7}$, Phys. Rev. Lett. 95, 127001 (2005).

[5] J. Leshen, M. Kavai, I. Giannakis, Y. Kaneko, Y. Tokura, S. Mukherjee, W. C. Lee, and P. Aynajian, Emergent charge order near the doping-induced Mott-insulating quantum phase transition in $\mathrm{Sr}_{3} \mathrm{Ru}_{2} \mathrm{O}_{7}$, Commun. Phys. 2, 36 (2019).

[6] S. I. Ikeda, S. I. Ikeda, Y. Maeno, Y. Maeno, S. Nakatsuji, M. Kosaka, and Y. Uwatoko, Ground state in $\mathrm{Sr}_{3} \mathrm{Ru}_{2} \mathrm{O}_{7}$ : Fermi liquid close to a ferromagnetic instability, Phys. Rev. B 62, R6089 (2000).

[7] L. Capogna, E. M. Forgan, S. M. Hayden, A. Wildes, J. A. Duffy, A. P. Mackenzie, R. S. Perry, S. Ikeda, Y. Maeno, and S. $\mathrm{P}$. Brown, Observation of two-dimensional spin fluctuations in the bilayer ruthenate $\mathrm{Sr}_{3} \mathrm{Ru}_{2} \mathrm{O}_{7}$ by inelastic neutron scattering, Phys. Rev. B 67, 012504 (2003).

[8] Q. Zhang, F. Ye, W. Tian, H. Cao, S. Chi, B. Hu, Z. Diao, D. A. Tennant, R. Jin, J. Zhang, and W. Plummer, Manganese-induced magnetic symmetry breaking and its correlation with the metalinsulator transition in bilayered $\mathrm{Sr}_{3}\left(\mathrm{Ru}_{1-x} \mathrm{Mn}_{x}\right)_{2} \mathrm{O}_{7}$, Phys. Rev. B 95, 220403(R) (2017).

[9] D. O. Brodsky, M. E. Barber, J. A. N. Bruin, R. A. Borzi, S. A. Grigera, R. S. Perry, A. P. Mackenzie, and C. W. Hicks, Strain and vector magnetic field tuning of the anomalous phase in $\mathrm{Sr}_{3} \mathrm{Ru}_{2} \mathrm{O}_{7}$, Sci. Adv. 3, e1501804 (2017).

[10] A. W. Rost, R. S. Perry, J. F. Mercure, A. P. Mackenzie, and S. A. Grigera, Entropy landscape of phase formation associated with quantum criticality in $\mathrm{Sr}_{3} \mathrm{Ru}_{2} \mathrm{O}_{7}$, Science 325, 1360 (2009).

[11] A. W. Rost, S. A. Grigera, J. A. N. Bruin, R. S. Perry, D. Tian, S. Raghu, S. A. Kivelson, and A. P. Mackenzie, Thermodynamics of phase formation in the quantum critical metal $\mathrm{Sr}_{3} \mathrm{Ru}_{2} \mathrm{O}_{7}$, Proc. Natl. Acad. Sci. USA 108, 16549 (2011).

[12] S. A. Grigera, Quantum critical points and nematics: the ruthenate $\mathrm{Sr}_{3} \mathrm{Ru}_{2} \mathrm{O}_{7}$, Acta. Crys. A64, C116 (2008).

[13] W. Wu, A. McCollam, S. A. Grigera, R. S. Perry, A. P. MacKenzie, and S. R. Julian, Quantum critical metamagnetism of $\mathrm{Sr}_{3} \mathrm{Ru}_{2} \mathrm{O}_{7}$ under hydrostatic pressure, Phys. Rev. B 83, 045106 (2011).

[14] G. Cao, S. McCall, J. Crow, and R. Guertin, Multiple magnetic phase transitions in single-crystal $\left(\mathrm{Sr}_{1-x} \mathrm{Ca}_{x}\right)_{3} \mathrm{Ru}_{2} \mathrm{O}_{7}$ for $0<x<1.0$, Phys. Rev. B 56, 5387 (1997).

[15] Y. Yoshida, S. I. Ikeda, H. Matsuhata, N. Shirakawa, C. H. Lee, and $\mathrm{S}$. Katano, Crystal and magnetic structure of $\mathrm{Ca}_{3} \mathrm{Ru}_{2} \mathrm{O}_{7}$, Phys. Rev. B 72, 054412 (2005).

[16] Z. Qu, L. Spinu, H. Yuan, V. Dobrosavljević, W. Bao, J. W. Lynn, M. Nicklas, J. Peng, T. Liu, D. Fobes, E. Flesch, and Z. Q. Mao, Unusual heavy-mass nearly ferromagnetic state with a surprisingly large Wilson ratio in the double layered ruthenates $\left(\mathrm{Sr}_{1-x} \mathrm{Ca}_{x}\right)_{3} \mathrm{Ru}_{2} \mathrm{O}_{7}$, Phys. Rev. B 78, 180407 (2008).

[17] Z. Qu, J. Peng, T. Liu, D. Fobes, V. Dobrosavljević, L. Spinu, and Z. Q. Mao, Effect of disorder on quantum phase transition in the double layered ruthenates $\left(\mathrm{Sr}_{1-x} \mathrm{Ca}_{x}\right)_{3} \mathrm{Ru}_{2} \mathrm{O}_{7}$, Phys. Rev. B 86, 014434 (2012)

[18] D. J. Singh and I. I. Mazin, Electronic structure and magnetism of $\mathrm{Sr}_{3} \mathrm{Ru}_{2} \mathrm{O}_{7}$, Phys. Rev. B 63, 165101 (2001).

[19] C. Dhital, T. Hogan, W. Zhou, X. Chen, Z. Ren, M. Pokharel, Y. Okada, M. Heine, W. Tian, Z. Yamani, C. Opeil, J. S. Helton, J. W. Lynn, Z. Wang, V. Madhavan, and S. D. Wilson, Carrier localization and electronic phase separation in a doped spin- orbit-driven Mott phase in $\mathrm{Sr}_{3}\left(\mathrm{Ir}_{1-x} \mathrm{Ru}_{x}\right)_{2} \mathrm{O}_{7}$, Nat. Commun. 5, 3377 (2014).

[20] J. F. Mitchell, J. E. Millburn, M. Medarde, S. Short, J. D. Jorgensen, and M. T. Fernandez-Diaz, $\mathrm{Sr}_{3} \mathrm{Mn}_{2} \mathrm{O}_{7}: \mathrm{Mn}^{4+}$ parent compound of the $n=2$ layered CMR manganites, J. Solid State Chem. 141, 599 (1998).

[21] D. Mesa, F. Ye, S. Chi, J. A. Fernandez-Baca, W. Tian, B. Hu, R. Jin, E. W. Plummer, and J. Zhang, Single-bilayer $E$-type antiferromagnetism in Mn-substituted $\mathrm{Sr}_{3} \mathrm{Ru}_{2} \mathrm{O}_{7}$ : neutron scattering study, Phys. Rev. B 85, 180410(R) (2012).

[22] J. R. L. de Almeida and D. J. Thouless, Stability of the Sherrington-Kirkpatrick solution of a spin glass model, J. Phys. A. Math. Gen. 11, 983 (1978).

[23] K. Binder and A. P. Young, Spin glasses-experimental facts, theoretical concepts, and open questions, Rev. Mod. Phys. 58, 801 (1986).

[24] J. A. Mydosh, Spin Glasses (CRC Press, London, 1993).

[25] J. A. Mydosh, Spin glasses: redux: an updated experimental/materials survey, Rep. Prog. Phys. 78, 052501 (2015).

[26] F. Wang, J. Kim, Y. J. Kim, and G. D. Gu, Spin-glass behavior in $\mathrm{LuFe}_{2} \mathrm{O}_{4+\delta}$, Phys. Rev. B 80, 024419 (2009).

[27] R. Nepal, Z. Wang, S. Dai, M. Saghayezhian, Y. Zhu, E. W. Plummer, and R. Jin, Emergent spin glass behavior created by self-assembled antiferromagnetic $\mathrm{NiO}$ columns in ferrimagnetic $\mathrm{NiFe}_{2} \mathrm{O}_{4}, \mathrm{ACS}$ Appl. Mater. Interfaces 12, 38788 (2020).

[28] J. Nogués and I. K. Schuller, Exchange bias, J. Magn. Magn. Mater. 192, 203 (1999).

[29] M. Ali, P. Adie, C. H. Marrows, D. Greig, B. J. Hickey, and R. L. Stamps, Exchange bias using a spin glass, Nat. Mater. 6, 70 (2007).

[30] C. Chen, J. Kim, V. B. Nascimento, Z. Diao, J. Teng, B. Hu, G. Li, F. Liu, J. Zhang, R. Jin, and E. W. Plummer, Hidden phases revealed at the surface of double-layered $\mathrm{Sr}_{3}\left(\mathrm{Ru}_{1-x} \mathrm{Mn}_{x}\right)_{2} \mathrm{O}_{7}$, Phys. Rev. B 94, 085420 (2016).

[31] Y. Yang, M. Fu, Q. Zou, Z. Gai, B. Hu, W. Xie, T. AlbrechtSchoenzart, J. Zhang, R. Jin, and E. W. Plummer, Surface critical point determined by octahedral tilt in $\mathrm{Sr}_{3}\left(\mathrm{Ru}_{1-x} \mathrm{Mn}_{x}\right)_{2} \mathrm{O}_{7}$, J. Electron Spectrosc. (2021), unpublished.

[32] H. Meskine, Z. S. Popović, and S. Satpathy, Electronic structure and exchange interaction in the layered perovskite $\mathrm{Sr}_{3} \mathrm{Mn}_{2} \mathrm{O}_{7}$, Phys. Rev. B 65, 094402 (2002).

[33] N. Mizutani, A. Kitazawa, N. Ohkuma, and M. Kato, Synthesis of strontium-manganese double oxides, Kogyo Kagaki Zasshi 73, 1097 (1970).

[34] L. J. Gillie, A. J. Wright, J. Hadermann, G. Van Tendeloo, and C. Greaves, Synthesis and characterization of the reduced double-layer manganite $\mathrm{Sr}_{3} \mathrm{Mn}_{2} \mathrm{O}_{6+x}$, J. Solid State Chem. 175, 188 (2003).

[35] R. Nepal, Q. Zhang, S. Dai, W. Tian, S. E. Nagler, and R. Jin, Structural and magnetic transitions in spinel $\mathrm{FeMn}_{2} \mathrm{O}_{4}$ single crystals, Phys. Rev. B 97, 024410 (2018).

[36] M. B. Salamon and M. Jaime, The physics of manganites: structure and transport, Rev. Mod. Phys. 73, 583 (2001).

[37] R. Bindu, G. Adhikary, S. K. Pandey, S. Patil, and K. Maiti, Spectral evolution in an insulator exhibiting linear specific heat, New J. Phys. 12, 033026 (2010).

[38] J. M. Schliesser and B. F. Woodfield, Lattice vacancies responsible for the linear dependence of the low-temperature heat capacity of insulating materials, Phys. Rev. B 91, 024109 (2015). 